\title{
Compostos bioativos obtidos de subprodutos vegetais com propriedades antimicrobianas
}

\author{
Nathana Lazzarotto Cristofoli, Francine Novack Victoria
}

https://doi.org/10.4322/mp.978-65-991393-3-8.c5

\begin{abstract}
Resumo
Frutas e vegetais são os produtos alimentares mais consumidos globalmente. In natura ou minimamente processados são ricos em nutrientes que auxiliam na saúde do consumidor. Bagaço, folhas, cascas e sementes são subprodutos com significativa quantidade de compostos bioativos, que desempenham atividade antimicrobiana, antiinflamatória, antioxidante, entre outras, sendo viável o uso de tecnologias existentes para o aproveitamento sustentável. A tendência atual de substituir os conservantes sintéticos despertou o interesse em procurar conservantes alternativos mais naturais que sejam eficientes contra uma gama de micro-organismos e que não prejudicam o meio ambiente tornando viável a exploração de subprodutos para esses fins. Esta revisão tem como objetivo reunir estudos que destaquem a atividade antimicrobiana obtida do aproveitamento de subprodutos vegetais, bem como discutir seu potencial como conservante de alimentos e os métodos de obtenção desses compostos naturais.
\end{abstract}

Palavras-chave: aditivo alimentar, extratos naturais, fitoquímicos, plantas medicinais, subproduto orgânico.

\section{Introdução}

O processamento de frutas e vegetais gera uma grande quantidade de subprodutos, como sementes e cascas, representando em média 30\% do total de produção, os quais são normalmente descartados como resíduos (FILHO; FRANCO, 2015; SAGAR et al., 2018). Porém, esses possuem em sua composição importantes compostos, que podem estar relacionados a melhoria e manutenção da qualidade da saúde humana (KIM et al., 2011; O'SHEA; ARENDT; GALLAGHER, 2012; WANG et al., 2007). O uso desses subprodutos como aditivo alimentar tem possibilitado evitar ou retardar a deterioração dos alimentos na forma de antioxidantes, corantes, aromatizantes, agentes espessantes e antimicrobianos. Sabe-se atualmente que os aditivos sintéticos comumente usados pela indústria de alimentos, tem despertado preocupação quanto a possível toxicidade, sendo comprovado em diversos estudos in vitro e in vivo o desenvolvimento de câncer devido a exposição excessiva e/ou prolongada a esses compostos (LIU; MABURY, 2020). Nesse contexto, há uma crescente demanda dos consumidores por alimentos que contenham menos aditivos sintéticos (DAVID; STEENSON; DAVIDSON, 2013; RIBEIRO et al., 2019).

As especiarias com fins medicinais são usadas há muitos anos e consideradas uma alternativa para a obtenção de extratos com propriedades antioxidantes e 
antimicrobianas usadas para preservar os alimentos (MARTIN et al., 2012; SZABO et al., 2010; YAHIA, 2017). O alho (Allium sativum), por exemplo, pode ser utilizado como aditivo natural, no qual sua casca apresenta substâncias biologicamente ativas capazes de inibir o crescimento de bactérias patogênicas, destacando a tecnologia de aproveitamento de resíduos agroindustriais como promissora (KALLEL et al., 2014). Esta revisão tem como objetivo reunir estudos que destaquem a atividade antimicrobiana obtida do aproveitamento de subprodutos vegetais, bem como discutir suas possíveis aplicações em alimentos e os métodos de obtenção desses compostos naturais.

\section{Compostos bioativos em subprodutos vegetais}

\subsection{Potencial biológico de subprodutos de origem vegetal}

Resíduos ou subprodutos hortícolas são as partes de uma matéria-prima vegetal sem valor comercial, descartadas desde a cadeia de abastecimento até o consumidor final (FAO, 2014). O crescimento populacional é um dos responsáveis pelo grande aumento de geração de resíduos em todo o mundo, seja através do consumo de alimentos in natura ou pelas indústrias processadoras (SAGAR et al., 2018). Esses resíduos são muitas vezes utilizados como ração animal, por conter nutrientes ou são eliminados no meio ambiente causando problemas ambientais e aumento nos custos do produto final (ANGULO et al., 2012). Bagaço, caule, folhas, cascas e sementes são exemplos de subprodutos, sendo os dois últimos os de maior disponibilidade e que tem apresentado extratos com bons resultados em relação à atividade antioxidante (O'SHEA; ARENDT; GALLAGHER, 2012; VAN DER GOOT et al., 2016).

Os subprodutos vegetais apresentam como potencial biológico atividade antioxidante e antimicrobiana, servindo como fonte de compostos bioativos (CHANDA et al., 2010; RUBILAR et al., 2007; SAGAR et al., 2018). Esses compostos estão presentes em pequenas quantidades nos alimentos e exercem efeito benéfico à saúde humana, como a redução do risco do desenvolvimento de doenças crônicas (ALMEIDA et al., 2011; YAHIA, 2017). Dentre eles estão os carotenoides e compostos fenólicos, que englobam os flavonoides, ácidos fenólicos e taninos (MATTILA et al., 2011).

Pesquisas têm sido realizadas a fim de desenvolver novos produtos com maior valor agregado, utilizando-os na indústria farmacêutica, alimentícia e de cosméticos (SAGAR et al., 2018; TREMOCOLDI et al., 2018; VAN DER GOOT et al., 2016; WANG et al., 2007). A recuperação de compostos bioativos e a utilização nas diversas áreas se faz necessária para diminuir o impacto ambiental e econômico. A Tabela 1 apresenta algumas das atividades biológicas relacionadas aos compostos bioativos de fontes vegetais.

Tabela 1. Atividade biológica de compostos bioativos obtidos de fontes vegetais.

\begin{tabular}{cccc}
\hline Atividade Biológica & Compostos & Fonte & Referência \\
\hline & $\begin{array}{c}\text { Flavonoides } \\
\text { (Resveratrol, } \\
\text { Quercetina) }\end{array}$ & $\begin{array}{c}\text { Subproduto de } \\
\text { goiaba, polpa } \\
\text { de acerola }\end{array}$ & $\begin{array}{c}\text { (DA SILVA et al., } \\
\text { 2014) }\end{array}$ \\
\cline { 2 - 4 } Antibacterino & $\begin{array}{c}\text { Composto fenólico } \\
\text { (Cumarina) }\end{array}$ & $\begin{array}{c}\text { Subproduto de } \\
\text { maracujá }\end{array}$ & \\
& $\begin{array}{c}\text { Flavonoide } \\
\text { (Floretina) }\end{array}$ & Maçã & \\
& & &
\end{tabular}




\begin{tabular}{|c|c|c|c|}
\hline & $\begin{array}{c}\text { Ácidos fenólicos, } \\
\text { flavonoides, } \\
\text { taninos }\end{array}$ & Berries & (CÔTÉ et al., 2011) \\
\hline Antiviral & $\begin{array}{c}\text { Flavonoides } \\
\text { (Flavanas) }\end{array}$ & Chás & (FRIEDMAN, 2007) \\
\hline \multirow[b]{3}{*}{ Antifúngico } & $\begin{array}{l}\text { Flavonoides } \\
\text { (Flavonas) }\end{array}$ & $\begin{array}{l}\text { Figo, amora, } \\
\text { jaca }\end{array}$ & (ARIF et al., 2009) \\
\hline & $\begin{array}{l}\text { Flavonoides } \\
\text { (Flavonol) }\end{array}$ & Própolis & (ARIF et al., 2009) \\
\hline & $\begin{array}{c}\text { Flavonoides } \\
\text { (Naringina, } \\
\text { hesperidina, neo- } \\
\text { hesperidina) }\end{array}$ & Citrus & $\begin{array}{c}\text { (SALAS et al., } \\
\text { 2011) }\end{array}$ \\
\hline \multirow{6}{*}{ Anti-inflamatório } & $\begin{array}{l}\text { Ácidos fenólicos } \\
\text { Flavonóides }\end{array}$ & $\begin{array}{c}\text { Cascas de } \\
\text { frutas cítricas }\end{array}$ & (FAVA et al., 2013) \\
\hline & $\begin{array}{l}\text { Flavonoides } \\
\text { (Catequina, } \\
\text { Isoflavona) }\end{array}$ & Chá verde, soja & $\begin{array}{c}\text { (SERGENT et al., } \\
\text { 2010) }\end{array}$ \\
\hline & $\begin{array}{l}\text { Flavonoides } \\
\text { (Catequina, } \\
\text { Epicatequina) }\end{array}$ & $\begin{array}{l}\text { Subproduto de } \\
\text { abacate }\end{array}$ & $\begin{array}{c}\text { (TREMOCOLDI et } \\
\text { al., 2018) }\end{array}$ \\
\hline & Polifenóis & Aveia & $\begin{array}{c}\text { (GUO; KONG; } \\
\text { MEYDANI, 2009) }\end{array}$ \\
\hline & $\begin{array}{l}\text { Flavonoides } \\
\text { (Quercetina) }\end{array}$ & $\begin{array}{l}\text { Cebola, chá, } \\
\text { maçã }\end{array}$ & $\begin{array}{c}\text { (CHEN; ZHOU; JI, } \\
\text { 2010) } \\
\end{array}$ \\
\hline & $\begin{array}{l}\text { Flavonoides } \\
\text { (resveratrol) }\end{array}$ & Vinho e berries & $\begin{array}{l}\text { (BISHT; WAGNER; } \\
\text { BULMER, 2010) }\end{array}$ \\
\hline \multirow[b]{4}{*}{ Anticancerígeno } & $\begin{array}{l}\text { Carotenoides } \\
\text { (Licopeno) }\end{array}$ & Tomate & (KONG et al., 2010) \\
\hline & $\begin{array}{l}\text { Compostos } \\
\text { fenólicos } \\
\text { (Catequina) }\end{array}$ & Chá verde & $\begin{array}{l}\text { (IGNAT; VOLF; } \\
\text { POPA, 2011) }\end{array}$ \\
\hline & Polifenóis & Aveia & $\begin{array}{c}\text { (GUO; KONG; } \\
\text { MEYDANI, 2009) }\end{array}$ \\
\hline & $\begin{array}{l}\text { Flavonoides } \\
\text { (Quercetina) }\end{array}$ & $\begin{array}{l}\text { Cebola, chá, } \\
\text { maçã }\end{array}$ & $\begin{array}{c}\text { (CHEN; ZHOU; Jl, } \\
\text { 2010; EKSTRÖM et } \\
\text { al., 2011) }\end{array}$ \\
\hline \multirow{5}{*}{$\begin{array}{c}\text { Efeitos } \\
\text { cardioprotetores }\end{array}$} & $\begin{array}{c}\text { Carotenoides } \\
\text { (Licopeno) }\end{array}$ & Tomate & (KONG et al., 2010) \\
\hline & $\begin{array}{l}\text { Compostos } \\
\text { fenólicos } \\
\text { (Catequina) } \\
\end{array}$ & Chá verde & $\begin{array}{l}\text { (IGNAT; VOLF; } \\
\text { POPA, 2011) }\end{array}$ \\
\hline & $\begin{array}{c}\text { Polifenol } \\
\text { (Resveratrol) }\end{array}$ & Vinho & $\begin{array}{l}\text { (GRESELE et al., } \\
\text { 2011) }\end{array}$ \\
\hline & Polifenóis & $\begin{array}{l}\text { Cacau, vinho, } \\
\text { semente de } \\
\text { uva, soja, romã }\end{array}$ & $\begin{array}{c}\text { (GHOSH; } \\
\text { SCHEEPENS, } \\
\text { 2009) }\end{array}$ \\
\hline & Polifenóis & Bagaço de uva & (FERRI et al., 2016) \\
\hline
\end{tabular}




\begin{tabular}{cc}
\hline Anti colesterol & $\begin{array}{c}\text { Flavonoides } \\
\text { Taninos } \\
\text { Antocianinas }\end{array}$ \\
\hline
\end{tabular}

Côte et al. (2011) comprovaram que a incorporação de antimicrobianos naturais extraídos de cramberry são eficientes para inibir Pseudomonas aeruginosa, Staphylococcus aureus e Escherichia coli, podendo ser usada como uma barreira adicional pela indústria de alimentos.

Salas et al. (2011) avaliaram o efeito da incorporação de extratos de Citrus, como a naringina, hesperidina e neo-hesperidina, em diferentes fungos (Aspergillus parasiticus, Aspergillus flavus, Fusarium semitectum e Penicillium expansum), observando efeito anfúngico.

Chen, Zhou e Ji (2010) relataram que a quercetina, proveniente de extratos de cebola, chá e maçã exibem ação antioxidativa, efeitos anti-inflamatórios e vasodilatadores, além de reduzir a indução de câncer de cólon em ratos.

A presença de compostos fenólicos (procianidina B1 e B2, ácido trans-5-O-cafeoil-Dquínico, epicatequina e catequina) com propriedades antioxidantes e antiinflamatórias na casca e semente do abacate apresentam potencial uso na indústria alimentícia e farmacêutica (TREMOCOLDI et al., 2018). Esses compostos também são relacionados ao potencial terapêutico, protegendo as células contra os danos causados pelos radicais livres, levando ao aumento da expectativa de vida devido a redução do risco de doenças (BISHT; WAGNER; BULMER, 2010).

Além das propriedades biológicas citadas na Tabela 1 , esses compostos podem contribuir inversamente com a obesidade e diabetes, além de distúrbios neurológicos (JOSEPH et al., 2009; SZKUDELSKA; SZKUDELSKI, 2010).

Os produtos e subprodutos de origem vegetal são atraentes fontes de compostos bioativos, apresentando diversificada atividade biológica que proporciona embasamento para pesquisas mais aprofundadas sobre o potencial desses extratos, bem como sua aplicação em outros produtos.

\section{Antimicrobianos naturais}

\subsection{Doenças Transmitidas por Alimentos}

Doenças Transmitidas por Alimentos (DTA) são adquiridas através da ingestão de alimentos contendo agentes contaminantes, como micro-organismos patogênicos, toxinas bacterianas ou produtos químicos, em quantidades que afetem a saúde do consumidor (BRASIL, 2010).

O aumento de patógenos resistentes a agentes antimicrobianos é um agravante que torna os tratamentos de doenças infecciosas ineficazes, sendo associado ao uso incorreto de antibióticos na medicina humana, veterinária e na agricultura (WHO, 2020). Como exemplo podemos citar o uso difundido de fluoroquinolonas em animais como aves e suínos, possibilitando o aparecimento de bactérias resistentes na alimentação humana (HSUEH et al., 2004).

\subsection{Atividade antimicrobiana de subprodutos vegetais}

Os agentes antimicrobianos são compostos químicos presentes naturalmente ou adicionados aos alimentos com a finalidade de inibir ou destruir micro-organismos 
patogênicos ou deteriorantes, garantindo assim a segurança microbiológica e evitando perdas (DAVID; STEENSON; DAVIDSON, 2013).

Os extratos obtidos a partir de materiais vegetais (folhas, sementes, cascas, raízes, óleos) apresentam atividade antimicrobiana para uma vasta gama de microorganismos e são relatados em diversos estudos científicos (AGOURRAM et al., 2013; AL-ZOREKY, 2009; ČANADANOVIĆ-BRUNET et al., 2011; CHUTIA et al., 2009; CRISTOFOLI et al., 2018; DU et al., 2009; ESPINA et al., 2011; FRIEDMAN, 2007; GALVÃO et al., 2010; HAYRAPETYAN; HAZELEGER; BEUMER, 2012; KALLEL et al., 2014; KANATT; CHANDER; SHARMA, 2010; KANATT; ARJUN; SHARMA, 2011; KATALINIĆ et al., 2010; KOOLEN et al., 2013; MAHMUD et al., 2009; MANDALARI et al., 2007, 2010; MARTIN et al., 2012; MARTINI et al., 2009; PERUMALLA; HETTIARACHCHY, 2011; SAGDIC et al., 2011; SUDJANA et al., 2009; TAVEIRA et al., 2010; WONGHIRUNDECHA; SUMPAVAPOL, 2012). Alguns destes são apresentados na Tabela 2.

Tabela 2. Subprodutos vegetais com atividade antimicrobiana.

\begin{tabular}{|c|c|c|}
\hline Subproduto & Micro-organismo & Referência \\
\hline $\begin{array}{l}\text { Bagaço de } \\
\text { azeitona (Olea } \\
\text { europaea) }\end{array}$ & $\begin{array}{c}\text { Escherichia coli, Salmonella enterica, } \\
\text { Listeria monocytogenes, Staphylococcus } \\
\text { aureus }\end{array}$ & $\begin{array}{l}\text { (FRIEDMAN, } \\
\text { 2007) }\end{array}$ \\
\hline $\begin{array}{c}\text { Bagaço de } \\
\text { goiaba (Psidium } \\
\text { guajava) }\end{array}$ & S. aureus, L. monocytogenes & $\begin{array}{l}\text { (MARTIN et al., } \\
\text { 2012) }\end{array}$ \\
\hline $\begin{array}{l}\text { Bagaço de uva } \\
\text { (Vitis vinífera, } \\
\text { Vitis labrusca) }\end{array}$ & $\begin{array}{l}\text { S. aureus, L. monocytogenes, Salmonella, } \\
\text { Enterococos, aeróbios mesófilos totais e } \\
\text { bactérias psicrotróficas, bolores e leveduras }\end{array}$ & $\begin{array}{l}\text { (MARTIN et al., } \\
\text { 2012; SAGDIC et } \\
\text { al., 2011) }\end{array}$ \\
\hline $\begin{array}{l}\text { Cascas de alho } \\
\text { (Allium sativum } \\
\text { L.) }\end{array}$ & $\begin{array}{c}\text { Klebsiella pneumoniae, Pseudomonas } \\
\text { aeruginosa, Bacillus thuringiensis, Bacillus } \\
\text { subtilis, S. aureus }\end{array}$ & $\begin{array}{l}\text { (KALLEL et al., } \\
\text { 2014) }\end{array}$ \\
\hline $\begin{array}{l}\text { Cascas de } \\
\text { amendoim } \\
\text { (Arachis } \\
\text { hypogaea) }\end{array}$ & S. aureus, L. monocytogenes & $\begin{array}{l}\text { (MARTIN et al., } \\
\text { 2012) }\end{array}$ \\
\hline $\begin{array}{c}\text { Casca de } \\
\text { bergamota } \\
\text { (Citrus bergamia } \\
\text { Risso) }\end{array}$ & $\begin{array}{l}\text { E. coli, Salmonella typhimurium, } \\
\text { Pseudomonas putida }\end{array}$ & $\begin{array}{l}\text { (MANDALARI et } \\
\text { al., 2007) }\end{array}$ \\
\hline $\begin{array}{l}\text { Casca de coco } \\
\text { (Cocos nucifera } \\
\text { Linn.) }\end{array}$ & L. monocytogenes, S. aureus, Vibrio cholera & $\begin{array}{l}\text { (WONGHIRUNDE } \\
\text { CHA; } \\
\text { SUMPAVAPOL, } \\
\text { 2012) }\end{array}$ \\
\hline $\begin{array}{l}\text { Casca de grão } \\
\text { de bico (Cicer } \\
\text { arietinum) }\end{array}$ & Bacillus cereus, S. aureus & $\begin{array}{l}\text { (KANATT; ARJUN; } \\
\text { SHARMA, 2011) }\end{array}$ \\
\hline $\begin{array}{l}\text { Casca de limão } \\
\text { (Citrus acida) }\end{array}$ & $\begin{array}{l}\text { B. subtilis, Bacillus cereus, S. aureus, E. coli, } \\
\text { Enterobacter aerogenes, S. Typhimurium, } \\
\text { Aspergillus ficuum, Aspergillus niger, } \\
\text { Aspergillus fumigatus, Aspergillus flavus, } \\
\text { Fusarium saloni, Fusarium oxysporum, } \\
\text { Pencillium digitatum, Candida utilis }\end{array}$ & $\begin{array}{l}\text { (MAHMUD et al., } \\
\text { 2009) }\end{array}$ \\
\hline $\begin{array}{l}\text { Casca de maçã } \\
\text { (Malus }\end{array}$ & $\begin{array}{l}\text { L. monocytogenes } \\
\text { S. aureus, Pseudomonas fluorescens }\end{array}$ & $\begin{array}{l}\text { (AGOURRAM et } \\
\text { al., 2013; DU et }\end{array}$ \\
\hline
\end{tabular}




\begin{tabular}{|c|c|c|}
\hline domestica) & & al., 2009) \\
\hline $\begin{array}{c}\text { Casca de } \\
\text { mangostão } \\
\text { (Garcinia } \\
\text { mangostana } \\
\text { Linn.) } \\
\end{array}$ & $\begin{array}{c}\text { B. cereus, L. monocytogenes, S. aureus, V. } \\
\text { cholerae }\end{array}$ & $\begin{array}{l}\text { (WONGHIRUNDE } \\
\text { CHA; } \\
\text { SUMPAVAPOL, } \\
\text { 2012) }\end{array}$ \\
\hline $\begin{array}{l}\text { Casca de } \\
\text { maracujá } \\
\text { (Passiflora } \\
\text { edulis) }\end{array}$ & Candida albicans & $\begin{array}{l}\text { (GALVÃO et al., } \\
\text { 2010) }\end{array}$ \\
\hline $\begin{array}{l}\text { Casca de romã } \\
\text { (Punica } \\
\text { granatum) }\end{array}$ & $\begin{array}{l}\text { L. monocytogenes, S. aureus, B. cereus, B. } \\
\text { subtilis, K. pneumoniae, E. coli, S. enteritidis, } \\
\text { Yersinia enterocolitica }\end{array}$ & $\begin{array}{c}\text { (AL-ZOREKY, } \\
2009 ; \\
\text { HAYRAPETYAN; } \\
\text { HAZELEGER; } \\
\text { BEUMER, 2012; } \\
\text { KANATT; } \\
\text { CHANDER; } \\
\text { SHARMA, 2010) }\end{array}$ \\
\hline $\begin{array}{l}\text { Casca da uva } \\
\text { (Vitis vinifera) }\end{array}$ & $\begin{array}{l}\text { S. aureus, B. cereus, E. coli, Salmonella } \\
\text { infantis, Campylobacter coli }\end{array}$ & $\begin{array}{c}\text { (KATALINIĆ et al., } \\
\text { 2010) }\end{array}$ \\
\hline $\begin{array}{l}\text { Folha de amora } \\
\text { (Rubus } \\
\text { ulmifolius) }\end{array}$ & Helicobacter pylori & $\begin{array}{l}\text { (MARTINI et al., } \\
\text { 2009) }\end{array}$ \\
\hline $\begin{array}{l}\text { Folha de buriti } \\
\text { (Mauritia } \\
\text { flexuosa L.f.) }\end{array}$ & S. aureus, $P$. aeruginosa & $\begin{array}{l}\text { (KOOLEN et al., } \\
\text { 2013) }\end{array}$ \\
\hline $\begin{array}{l}\text { Folha de cajá } \\
\text { (Spondias } \\
\text { mombin) }\end{array}$ & $\begin{array}{l}\text { B. cereus, L. innocua, S. aureus, E. coli, } \\
\text { Saccharomyces cerevisiae }\end{array}$ & $\begin{array}{l}\text { (CRISTOFOLI et } \\
\text { al., 2018) }\end{array}$ \\
\hline $\begin{array}{l}\text { Folhas de caju } \\
\text { (Anacardium } \\
\text { occidentale) }\end{array}$ & $\begin{array}{c}\text { B. cereus, L. monocytogenes, S. aureus, E. } \\
\text { coli, S. Typhimurium, V. cholera }\end{array}$ & $\begin{array}{l}\text { (WONGHIRUNDE } \\
\text { CHA; } \\
\text { SUMPAVAPOL, } \\
\text { 2012) }\end{array}$ \\
\hline $\begin{array}{c}\text { Folhas de oliveira } \\
\text { (Olea europaea) }\end{array}$ & Campylobacter jejuni, H. pylori, S. aureus & $\begin{array}{l}\text { (SUDJANA et al., } \\
\text { 2009) }\end{array}$ \\
\hline $\begin{array}{l}\text { Folha de videira } \\
\text { (Vitis vinifera L.) }\end{array}$ & $\begin{array}{c}\text { S. aureus, B. cereus, C. jejuni, E. coli e } \\
\text { Salmonella infantis }\end{array}$ & $\begin{array}{c}\text { (KATALINIĆ et al., } \\
\text { 2010) }\end{array}$ \\
\hline $\begin{array}{l}\text { Óleo da casca de } \\
\text { tangerina (Citrus } \\
\text { reticulata) }\end{array}$ & $\begin{array}{c}\text { Alternaria alternata, Rhizoctonia } \\
\text { solani, Curvularia lunata, Fusarium } \\
\text { oxysporum, Helminthosporium oryzae }\end{array}$ & $\begin{array}{l}\text { (CHUTIA et al., } \\
\text { 2009) }\end{array}$ \\
\hline $\begin{array}{c}\text { Óleos essenciais } \\
\text { de laranja (Citrus } \\
\text { sinensis), limão } \\
\text { (Citrus limon) e } \\
\text { tangerina (Citrus } \\
\text { reticulata) }\end{array}$ & $\begin{array}{l}\text { Enterococcus faecium, S. aureus, } P . \\
\text { aeruginosa, S. enterica, E. coli, } \\
\text { L. monocytogenes }\end{array}$ & $\begin{array}{l}\text { (ESPINA et al., } \\
\text { 2011) }\end{array}$ \\
\hline $\begin{array}{l}\text { Pele de amêndoa } \\
\text { (Prunus dulcis) }\end{array}$ & L. monocytogenes, S. aureus & $\begin{array}{l}\text { (MANDALARI et } \\
\text { al., 2010) }\end{array}$ \\
\hline $\begin{array}{l}\text { Raiz de } \\
\text { beterraba (Beta } \\
\text { vulgaris) }\end{array}$ & S. aureus, B. cereus E. coli, $P$. aeruginosa & $\begin{array}{c}\text { (DJILAS; } \\
\text { MARKOV, 2011) }\end{array}$ \\
\hline
\end{tabular}




\begin{tabular}{|c|c|c|}
\hline $\begin{array}{l}\text { Semente de } \\
\text { maracujá } \\
\text { (Passiflora } \\
\text { edulis) }\end{array}$ & C. albicans & $\begin{array}{c}\text { (GALVÃO et al., } \\
\text { 2010) }\end{array}$ \\
\hline $\begin{array}{l}\text { Semente de } \\
\text { tomate (Solanum } \\
\text { lycopersicum) }\end{array}$ & $\begin{array}{c}\text { S. aureus, Staphylococcus epidermidis, } \\
\text { Micrococcus luteus, E. faecalis, B. cereus, C. } \\
\text { albicans }\end{array}$ & $\begin{array}{c}\text { (TAVEIRA et al., } \\
\text { 2010) }\end{array}$ \\
\hline $\begin{array}{l}\text { Semente de uva } \\
\text { (Vitis vinífera, } \\
\text { Vitis labrusca) }\end{array}$ & $\begin{array}{c}\text { L. monocytogenes, S. Typhimurium, E. coli, } \\
\text { Campylobacter jejuni } \\
\text { S. aureus } \\
\text { Pseudomonas spp., P. fluorescens, } \\
\text { Shewanella putrefaciens, Enterobacteriaceae } \\
\text { family }\end{array}$ & $\begin{array}{c}\text { (PERUMALLA; } \\
\text { HETTIARACHCH, } \\
\text { 2011) } \\
\text { (MARTIN et al., } \\
\text { 2012) } \\
\text { (KAKAEl; } \\
\text { SHAHBAZI, 2016) }\end{array}$ \\
\hline $\begin{array}{l}\text { Talos de } \\
\text { beterraba (Beta } \\
\text { vulgaris) }\end{array}$ & S. aureus, L. monocytogenes & $\begin{array}{c}\text { (MARTIN et al., } \\
\text { 2012) }\end{array}$ \\
\hline
\end{tabular}

Kallel et al. (2014) estudaram a atividade antimicrobiana de extratos de casca de alho (Allium sativum L.) e demonstraram que o extrato inibiu o crescimento de bactérias patogênicas. Essa propriedade pode ser atribuída aos compostos fenólicos identificados nos extratos, tais como ácido cafeico, p-cumárico, ferúlico e di-ferúlico.

Extratos de folhas de amora (Rubus ulmifolius) e os seus principais componentes foram testados quanto à sua atividade antibacteriana contra Helicobacter pylori. $\mathrm{O}$ extrato mostrou um elevado teor de ácido elágico, quercetina 3-O- $\beta$-d-glucopyranoside e rutina. A Concentração Bactericida Mínima (CBM) para o extrato da folha após $48 \mathrm{~h}$ de exposição foi de $134 \mu \mathrm{g} / \mathrm{mL}$. Para os compostos isolados, após $48 \mathrm{~h}$, o ácido elágico e campferol apresentaram as menores concentrações, 2 e $6 \mu \mathrm{gg} / \mathrm{mL}$, respectivamente (MARTINI et al., 2009). Os efeitos antibacterianos estão relacionados com diferentes propriedades biológicas (alvos celulares) sendo específicos para cada composto. A CBM para o extrato da folha de amora pode ser explicado devido a baixa concentração dos compostos individuais no extrato e a presença de outros componentes que podem não ter atividade antibacteriana.

Extratos da folha de cajá apresentaram concentração mínima inibitória (CMI) para a $E$. coli e S. cerevisiae de 15,6 e $250 \mu \mathrm{g} / \mathrm{mL}$, respectivamente, sendo o primeiro estudo com esta matéria-prima a apresentar inibição para levedura. Os solventes orgânicos permitiram a extração do ácido cafeico, elágico, gálico, catequina e rutina, enquanto o fluido supercrítico permitiu a obtenção do ácido elágico, gálico, vanílico e catequina, ressaltando a importância da tecnologia utilizada para obtenção de extratos de acordo com a atividade biológica desejada (CRISTOFOLI et al., 2018).

A atividade antimicrobiana de extratos ricos em flavonoides a partir da pele de amêndoa e casca de bergamota foi avaliada e comparada com os compostos orgânicos puros de cada subproduto. Extratos obtidos da pele de amêndoa foram inibitórios para $L$. monocytogenes, $S$. aureus e $S$. enterica ssp. enterica ser. Typhimurium nas concentrações de 250, 250 e $500 \mu \mathrm{g} / \mathrm{mL}$, respectivamente. Entre os compostos puros, a naringenina apresentou a maior atividade com concentração mínima de 250-500 $\mu \mathrm{g} / \mathrm{mL}$, seguido de epicatequina, ácido protocatecuico, catequina e Isoramnetina-3- $\mathrm{O}$-glucoside, estes dois últimos inibindo apenas L. monocytogenes e S. enterica, respectivamente (MANDALARI et al., 2010). Já os extratos da casca de bergamota apresentaram inibição do crescimento das bactérias Gram-negativas (E. coli, S. enterica ser. Typhimurium, P. putida). Em relação aos 
compostos puros o eriodictiol foi o mais ativo e inibiu as bactérias Gram-negativas já citadas, além de $B$. subtilis, $L$. innocua, L. lactis, S. aureus e $S$. cerevisiae com concentração mínima inibitória na faixa de 250 a $800 \mu \mathrm{g} / \mathrm{mL}$. O composto naringenina foi o segundo mais eficaz (MANDALARI et al., 2007).

Martin et al. (2012) avaliando a concentração mínima inibitória (CMI) e a concentração bactericida mínima (CBM) de subprodutos contra S. aureus e L. monocytogenes. Os extratos etanólico e metanólico de casca de amendoim (Arachis hypogaea) apresentaram os menores valores para $S$. aureus, com valores de 0,78 e 1,56 mg/mL para CMI e CBM, respectivamente. Para L. monocytogenes o extrato etanólico de bagaço de goiaba (Psidium guajava) obteve o menor CMI $(1,56 \mathrm{mg} / \mathrm{mL})$ embora tenha apresentado potencial bactericida reduzido com elevado valor de CBM $(12,5 \mathrm{mg} / \mathrm{mL})$.

Extratos da casca de grão de bico (Cicer arietinum) na concentração de $0,1 \%$ inibiram completamente $B$. cereus (KANATT; ARJUN; SHARMA, 2011). Já o extrato da casca de romã (Punica granatum) apresentou boa atividade antimicrobiana contra $S$. aureus e $B$. cereus possuindo CMI de 0,01\% (KANATT; CHANDER; SHARMA, 2010). Esses resultados devem estar relacionados à maior quantidade de taninos, flavonoides $\mathrm{e}$ glicosídeos presentes no extrato metanólico da casca de romã e mostram que extratos obtidos de subprodutos vegetais são eficientes conservantes naturais.

\section{Métodos de obtenção de antimicrobianos vegetais}

O processo de extração dos compostos bioativos de resíduos de alimentos pode ser realizado por diversas metodologias. Essas têm o objetivo de não afetar as propriedades funcionais dos compostos e manter os padrões de qualidade e segurança. A substância a ser extraída e o rendimento do processo são os principais itens a serem observados na escolha da metodologia mais apropriada, assim como 0 solvente utilizado (CRISTOFOLI et al., 2018; GIL-CHÁVEZ et al., 2013).

Entre as técnicas convencionais de extração temos o Soxhlet, que é uma extração simples e requer pouco treinamento. Diversos tipos de solventes podem ser usados nesse processo que apresenta como vantagem o baixo custo do equipamento sendo possível realizar extrações simultâneas (LUQUE DE CASTRO; PRIEGO-CAPOTE, 2010). Como desvantagens desse processo têm-se o longo tempo de extração e o uso de temperaturas elevadas, podendo resultar na decomposição de compostos termolábeis, além do uso elevado de solventes, sendo necessária uma etapa posterior para eliminação deste, podendo ainda, obter extratos não livres de solventes (LUQUE DE CASTRO; PRIEGO-CAPOTE, 2010).

Tecnologias não térmicas apresentam vantagens quanto aos processos convencionais, otimizando a eficiência do processamento (GAÑÁN; BRIGNOLE, 2011), pois proporcionam maior seletividade, menor tempo de extração e uso de baixas temperaturas, preservando a qualidade de compostos termo sensíveis, além de não utilizar solventes orgânicos tóxicos (DÍAZ-REINOSO et al., 2006; HERRERO et al., 2010).

\section{Possibilidade de aplicação dos extratos de subprodutos}

Estudos científicos com compostos antimicrobianos mostram que esses apresentam uma alternativa emergente para a conservação dos alimentos.

Osaili et al. (2021) reportaram que a adição de especiarias (sal, pimenta, gengibre, jalapenho, limão e alho) e óleos essenciais (carvacrol, cinamaldeído e timol) em peito 
de frango foi capaz de reduzir o número de Salmonella e E. coli durante o armazenamento a 4 e $10{ }^{\circ} \mathrm{C}$. Esse comportamento está relacionado com as propriedades antimicrobianas desses ingredientes.

Abdalla et al. (2007) estudando o efeito antimicrobiano do extrato de sementes de manga (Mangifera indica) em leite de vaca cru constataram que, após $6 \mathrm{~h}$ de incubação a $25^{\circ} \mathrm{C}$, a adição de 3000 ppm do extrato resultou na redução da contagem bacteriana de $9,9 \times 10^{8}$ para $5,0 \times 10^{3} \mathrm{UFC} / \mathrm{mL}$, enquanto que o crescimento de coliformes foi completamente inibida.

O extrato da casca de romã (Punica granatum) foi adicionado em pasta de carne e foi avaliado o efeito inibidor contra $L$. monocytogenes. A partir de uma contagem inicial de $3,5 \mathrm{log}$ UFC/g, o extrato da romã armazenado a $4{ }^{\circ} \mathrm{C}$ permitiu o crescimento de $1,5 \mathrm{log}$ ao fim do experimento (46 dias), enquanto que o controle atingiu 9,2 log no dia 18, demonstrando que o extrato pode servir como um aditivo com ação dupla, incorporando atividade antioxidante para a manutenção da cor do produto e exercendo efeito antibacteriano (HAYRAPETYAN; HAZELEGER; BEUMER, 2012).

Em estudo com extrato da casca de romã (Punica granatum) adicionado em produtos à base de frango foi comprovado o aumento da vida útil de 7 para 20 dias durante 0 armazenamento refrigerado (KANATT; CHANDER; SHARMA, 2010).

Al-Zoreky (2009) avaliou a eficácia do extrato da casca de romã contra $L$. monocytogenes em pedaços de peixe fresco. Os resultados revelaram que o extrato impediu a proliferação do patógeno, onde o número de células permaneceu abaixo do nível inoculado, 3 log UFC/g, até o fim do armazenamento (dia 6).

Em outro estudo, a adição de extrato de semente de uva em filmes de colza proteínagelatina inibiu o crescimento de $E$. coli e L. monocytogenes após 14 dias de armazenamento (JANG; SHIN; SONG, 2011).

Os óleos essenciais de bergamota, limão e chá verde quando incorporados à filmes de polissacarídeos inibiram o crescimento bacteriano de $L$. monocytogenes, E. coli e $S$. aureus durante 12 dias, comprovando sua eficácia nesse tipo de produto (SÁNCHEZGONZÁLEZ et al., 2011). Além disso, filmes e revestimentos comestíveis ajudaram a reduzir as preocupações ambientais criados por embalagens de plástico convencional. Filmes de quitosana incorporado com extrato de semente de uva apresentaram efeitos benéficos no retardo da oxidação lipídica e crescimento de Listeria monocytogenes em truta refrigerada a $4{ }^{\circ} \mathrm{C}$, auxiliando na mantenção da qualidade do produto e aumento da sua vida útil (KAKAEI; SHAHBAZI, 2016).

Extratos com propriedades antimicrobianas também são incorporados em sachês que são adicionados às embalagens normalmente utilizadas ou são aplicados diretamento em biofilmes para a preservação de carne, peixe, aves, pães, queijos, frutas e produtos hortícolas (CHIU; LAI, 2010; DA SILVEIRA et al., 2014; HOSSEINI et al., 2020; OSAILI et al., 2021; SHIEKH; BENJAKUL; SAE-LEAW, 2019; TIWARI et al., 2009; ZINOVIADOU; KOUTSOUMANIS; BILIADERIS, 2009).

A utilização de compostos bioativos a partir de resíduos vegetais em outros alimentos serve como conservante natural, preservando e aumentando a vida útil dos produtos, além de contribuir para a redução de perdas pós-colheita e incrementar a economia do país. 


\section{Considerações finais}

A recuperação de compostos bioativos de resíduos vegetais se torna pertinente, uma vez que esses produtos são obtidos de fontes naturais e econômicas que não teriam mais utilidade, contribuindo para a redução de danos ao meio ambiente e possibilitando a conservação de outros alimentos através de um aditivo natural. Fazem-se necessárias mais pesquisas que possam abranger outros compostos presentes nestes alimentos, como fibras e vitaminas explorando novas possibilidades de uso na indústria alimentar, farmacéutica, cosmética e os benefícios destes compostos na saúde humana.

\section{Referências}

ABDALLA, A. E. M. et al. Egyptian mango by-product 2: Antioxidant and antimicrobial activities of extract and oil from mango seed kernel. Food Chemistry, v. 103, n. 4, p. 1141-1152, 2007. https://doi.org/10.1016/j.foodchem.2006.10.026.

AgOURRAM, A. et al. Phenolic content, antioxidant potential, and antimicrobial activities of fruit and vegetable by-product extracts. International Journal of Food $\begin{array}{llllll}\text { Properties, } & \text { v. } 16, \quad \text { n. } 5, \quad \text { p. 1092-1104, }\end{array}$ https://doi.org/10.1080/10942912.2011.576446.

AL-ZOREKY, N. S. Antimicrobial activity of pomegranate (Punica granatum L.) fruit peels. International Journal of Food Microbiology, v. 134, n. 3, p. 244-248, 2009. https://doi.org/10.1016/j.ijfoodmicro.2009.07.002.

ALMEIDA, M. M. B. et al. Bioactive compounds and antioxidant activity of fresh exotic fruits from northeastern Brazil. Food Research International, v. 44, n. 7, p. 21552159, 2011. https://doi.org/10.1016/j.foodres.2011.03.051.

ANGULO, J. et al. Nutritional evaluation of fruit and vegetable waste as feedstuff for diets of lactating Holstein cows. Journal of Environmental Management, v. 95, n. SUPPL., p. S210-S214, 2012. https://doi.org/10.1016/j.jenvman.2011.06.050.

ARIF, T. et al. Natural products - Antifungal agents derived from plantsJournal of Asian Natural Products Research. Journal of Asian Natural Products Research, v. 11, p. 631-638, 2009. https://doi.org/10.1080/10286020902942350.

BISHT, K.; WAGNER, K. H.; BULMER, A. C. Curcumin, resveratrol and flavonoids as anti-inflammatory, cyto- and DNA-protective dietary compounds. Toxicology, v. 278, n. 1, p. 88-100, 2010. https://doi.org/10.1016/j.tox.2009.11.008.

BRASIL. Manual integrado de vigilância, prevenção e controle de doenças transmitidas por alimentos. Ministério da Saúde, Secretaria de Vigilância em Saúde, Departamento de Vigilância Epidemiológica. Brasília.

ČANADANOVIĆ-BRUNET, J. M. et al. Antioxidant and antimicrobial activities of beet root pomace extracts. Czech Journal of Food Science, v. 29, n. 6, p. 575-585, 2011. https://doi.org/10.17221/210/2010-CJFS.

CHANDA, S. et al. Fruit and vegetable peels - strong natural source of antimicrobics. In: MENDEZ-VILAS (org.). Current research, technology and education topics in applied microbiology and microbial biotechnology. Badajoz: FORMATEX Research Center, 2010. p. 444-450. 
CHEN, C.; ZHOU, J.; JI, C. Quercetin: A potential drug to reverse multidrug resistanceLife Sciences, Life Science, v.87, n. 11-12, p.333-338, 2010. https://doi.org/10.1016/j.Ifs.2010.07.004.

CHIU, P. E.; LAI, L. S. Antimicrobial activities of tapioca starch/decolorized hsian-tsao leaf gum coatings containing green tea extracts in fruit-based salads, romaine hearts and pork slices. International Journal of Food Microbiology, v. 139, n. 1-2, p. 2330, 2010. https://doi.org/10.1016/j.ijfoodmicro.2010.01.010.

CHUTIA, M. et al. Antifungal activity and chemical composition of Citrus reticulata Blanco essential oil against phytopathogens from North East India. LWT - Food Science and Technology, v. 42, n. 3, p. 777-780, 2009. https://doi.org/10.1016/j.Iwt.2008.09.015.

CÔTÉ, J. et al. Antimicrobial effect of cranberry juice and extracts. Food Control, v. 22, n. 8, p. 1413-1418, 2011. https://doi.org/10.1016/j.foodcont.2011.02.024.

CRISTOFOLI, N. L. et al. Antioxidant and antimicrobial potential of cajazeira leaves (Spondias mombin) extracts. Separation Science and Technology, v. 54, n. 4, p. 580-590, 2018. https://doi.org/10.1080/01496395.2018.1508233.

DA SILVA, L. M. R. et al. Quantification of bioactive compounds in pulps and byproducts of tropical fruits from Brazil. Food Chemistry, v. 143, p. 398-404, 2014. https://doi.org/10.1016/j.foodchem.2013.08.001.

DA SILVEIRA, S. M. et al. Chemical composition and antibacterial activity of Laurus nobilis essential oil towards foodborne pathogens and its application in fresh Tuscan sausage stored at $7^{\circ} \mathrm{C}$. LWT - Food Science and Technology, v. 59, n. 1, p. 86-93, 2014. https://doi.org/10.1016/j.Iwt.2014.05.032.

DAVID, J. R. D.; STEENSON, L. R.; DAVIDSON, P. M. Expectations and applications of natural antimicrobials to foods: A guidance document for users, suppliers, research and development, and regulatory agencies. Food Protection Trends, v. 33, n. 4, p. 241-250, 2013.

DÍAZ-REINOSO, B. et al. Supercritical $\mathrm{CO}_{2}$ extraction and purification of compounds with antioxidant activity. Journal of Agricultural and Food Chemistry, v. 54, n. 7, p. 2441-2469, 2006. https://doi.org/10.1021/jf052858j.

DU, Y. et al. Preparation of water-soluble chitosan from shrimp shell and its antibacterial activity. Innovative Food Science and Emerging Technologies, v. 10, n. 1, p. 103-107, 2009. https://doi.org/10.1016/j.ifset.2008.07.004.

EKSTRÖM, A. M. et al. Dietary quercetin intake and risk of gastric cancer: Results from a population-based study in Sweden. Annals of Oncology, v. 22, n. 2, p. 438-443, 2011. https://doi.org/10.1093/annonc/mdq390.

ESPINA, L. et al. Chemical composition of commercial citrus fruit essential oils and evaluation of their antimicrobial activity acting alone or in combined processes. Food Control, v. 22, n. 6, p. 896-902, 2011. https://doi.org/10.1016/j.foodcont.2010.11.021.

FAO. Definitional Framework Of Food Loss Global Initiative on Food Loss and Waste Reduction. Rome, Italy. 2014. Disponível em: www.fao.org/publications. Acesso em: 11 nov. 2020. 
FAVA, F. et al. New advances in the integrated management of food processing byproducts in Europe: Sustainable exploitation of fruit and cereal processing by-products with the production of new food products (NAMASTE EU). New Biotechnology, v. 30, n. 6, p. 647-655, 2013. https://doi.org/10.1016/j.nbt.2013.05.001.

FERRI, M. et al. Recovery of polyphenols from red grape pomace and assessment of their antioxidant and anti-cholesterol activities. New Biotechnology, v. 33, n. 3, p. 338-344, 2016. https://doi.org/10.1016/j.nbt.2015.12.004.

FILHO, W. B.do N.; FRANCO, C. R. Avaliação do potencial dos resíduos produzidos através do processamento agroindustrial no Brasil. Revista Viirtual de Química, v. 7, n. 6, 2015. https://doi.org/10.5935/1984-6835.20150116.

FRIEDMAN, M. Overview of antibacterial, antitoxin, antiviral, and antifungal activities of tea flavonoids and teas Molecular Nutrition and Food Research, v. 51, p. 116-134, 2007. https://doi.org/10.1002/mnfr.200600173.

GALVÃO, K. C. S. et al. Análise da atividade inibitória de Passiflora edulis Sims f. flavicarpa sobre Candida albicans. Revista Biociências, v. 16, p. 80-85, 2010.

GAÑÁN, N.; BRIGNOLE, E. A. Fractionation of essential oils with biocidal activity using supercritical CO2 - Experiments and modeling. Journal of Supercritical Fluids, v. 58, n. 1, p. 58-67, 2011. https://doi.org/10.1016/j.supflu.2011.04.010.

GHOSH, D.; SCHEEPENS, A. Vascular action of polyphenols. Molecular Nutrition and Food Research, v.53, p. 322-331, 2009. https://doi.org/10.1002/mnfr.200800182.

GIL-CHÁVEZ, G.J. et al. Technologies for extraction and production of bioactive compounds to be used as nutraceuticals and food Ingredients: An Overview. Comprehensive Reviews in Food Science and Food Safety, v. 12, n. 1, p. 5-23, 2013. https://doi.org/10.1111/1541-4337.12005.

GRESELE, P. et al. Effects of resveratrol and other wine polyphenols on vascular function: An update. Journal of Nutritional Biochemistry, v.22, p. 201-211, 2011. https://doi.org/10.1016/j.jnutbio.2010.07.004.

GUO, W.; KONG, E. H.; MEYDANI, M. Dietary polyphenols, inflammation, and cancer. $\begin{array}{lllll}\text { Nutrition and cancer, } & \text { v. 61, p. 807-810, }\end{array}$ https://doi.org/10.1080/01635580903285098.

HAYRAPETYAN, H.; HAZELEGER, W. C.; BEUMER, R. R. Inhibition of Listeria monocytogenes by pomegranate (Punica granatum) peel extract in meat paté at different temperatures. Food Control, v. 23, n. 1, p. 66-72, 2012. https://doi.org/10.1016/j.foodcont.2011.06.012.

HERRERO, M. et al. Supercritical fluid extraction: Recent advances and applications. Journal of Chromatography A, v. 1217, n. 16, p. 2495-2511, 2010. https://doi.org/10.1016/j.chroma.2009.12.019.

HOSSEINI, M. et al. Effect of sodium alginate coating containing clove (Syzgium Aromaticum) and lemon verbena (Aloysia Citriodora) essential oils and different packaging treatments on shelf life extension of refrigerated chicken breast. Journal of Food Processing and Preservation, 2020. Disponível em: 
https://onlinelibrary.wiley.com/doi/10.1111/jfpp.14946. Acesso em: 11 nov. 2020. https://doi.org/10.1111/jfpp.14946.

HSUEH, P. R. et al. Ciprofloxacin-resistant Salmonella enterica Typhimurium and Choleraesuis from pigs to humans, Taiwan. Emerging Infectious Diseases, v. 10, n. 1, p. 60-68, 2004. https://doi.org/10.3201/eid1001.030171.

IGNAT, I.; VOLF, I.; POPA, V. I. A critical review of methods for characterisation of polyphenolic compounds in fruits and vegetables. Food Chemistry, v.126, p. 18211835, 2011. https://doi.org/10.1016/j.foodchem.2010.12.026.

JANG, S-A.; SHIN, Y-J.; SONG, K. B. Effect of rapeseed protein-gelatin film containing grapefruit seed extract on "Maehyang" strawberry quality. International Journal of Food Science \& Technology, v. 46, n. 3, p. 620-625, 2011. https://doi.org/10.1111/j.1365-2621.2010.02530.x.

JOSEPH, J. et al. Nutrition, brain aging, and neurodegeneration. In: JOURNAL OF NEUROSCIENCE 2009, Anais [...]. : J Neurosci, 2009. p. 12795-12801. Disponível em: https://pubmed.ncbi.nlm.nih.gov/19828791/. Acesso em: 14 nov. 2020. https://doi.org/10.1523/JNEUROSCI.3520-09.2009.

KAKAEI, S.; SHAHBAZI, Y. Effect of chitosan-gelatin film incorporated with ethanolic red grape seed extract and Ziziphora clinopodioides essential oil on survival of Listeria monocytogenes and chemical, microbial and sensory properties of minced trout fillet. LWT - Food Science and Technology, v. 72, p. 432-438, 2016. https://doi.org/10.1016/j.Iwt.2016.05.021.

KALLEL, F. et al. Garlic (Allium sativum L.) husk waste as a potential source of phenolic compounds: Influence of extracting solvents on its antimicrobial and antioxidant properties. Industrial Crops and Products, v. 62, p. 34-41, 2014. https://doi.org/10.1016/j.indcrop.2014.07.047.

KANATT, S. R.; CHANDER, R.; SHARMA, A. Antioxidant and antimicrobial activity of pomegranate peel extract improves the shelf life of chicken products. International Journal of Food Science \& Technology, v. 45, n. 2, p. 216-222, 2010. https://doi.org/10.1111/j.1365-2621.2009.02124.x.

KANATT, S. R.; ARJUN, K.; SHARMA, A. Antioxidant and antimicrobial activity of legume hulls. Food Research International, v. 44, n. 10, p. 3182-3187, 2011. https://doi.org/10.1016/j.foodres.2011.08.022.

KATALINIĆ, V. et al. Polyphenolic profile, antioxidant properties and antimicrobial activity of grape skin extracts of 14 Vitis vinifera varieties grown in Dalmatia (Croatia).

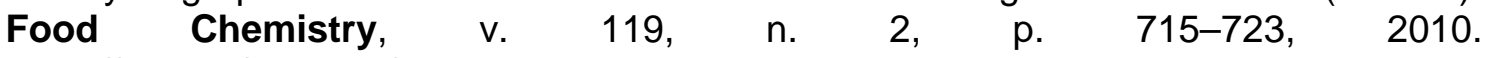
https://doi.org/10.1016/j.foodchem.2009.07.019.

$\mathrm{KIM}$, II S. et al. Antioxidant activities of hot water extracts from various spices. International Journal of Molecular Sciences, v. 12, n. 6, p. 4120-4131, 2011. https://doi.org/10.3390/ijms12064120.

KONG, K. W. et al. Revealing the power of the natural red pigment lycopene. Molecules, v. 15, n. 2, p. 959-987, 2010. https://doi.org/10.3390/molecules15020959.

KOOLEN, H. H. F. et al. Antioxidant, antimicrobial activities and characterization of phenolic compounds from buriti (Mauritia flexuosa L. f.) by UPLC-ESI-MS/MS. Food $\begin{array}{lllllll}\text { Research International, } & \text { v. 51, n. 2, p. 467-473, }\end{array}$ 
https://doi.org/10.1016/j.foodres.2013.01.039.

LEE, J. H. et al. Apple flavonoid phloretin inhibits Escherichia coli O157:H7 biofilm formation and ameliorates colon inflammation in rats. Infection and Immunity, v. 79, n. 12, p. 4819-4827, 2011. https://doi.org/10.1128/IAI.05580-11.

LIU, R.; MABURY, S. A. Synthetic phenolic antioxidants: A review of environmental occurrence, fate, human exposure, and toxicity. Environmental science \& $\begin{array}{llllll}\text { technology, } & \text { v. } 54, \quad \text { n. } 19, \quad \text { p. } 11706-11719,\end{array}$ https://doi.org/10.1021/acs.est.0c05077.

LUQUE DE CASTRO, M. D.; PRIEGO-CAPOTE, F. Soxhlet extraction: Past and present panacea. Journal of Chromatography A, v. 1217, n. 16, p. 2383-2389, 2010. https://doi.org/10.1016/j.chroma.2009.11.027.

MAHMUD, S. et al. Volatile components, antioxidant and antimicrobial activity of Citrus acida var. sour lime peel oil. Journal of Saudi Chemical Society, v. 13, n. 2, p. 195198, 2009. https://doi.org/10.1016/j.jscs.2009.03.001.

MANDALARI, G. et al. Antimicrobial activity of flavonoids extracted from bergamot (Citrus bergamia Risso) peel, a byproduct of the essential oil industry. Journal of Applied Microbiology, v. 103, n. 6, p. 2056-2064, 2007. https://doi.org/10.1111/j.1365-2672.2007.03456.x.

MANDALARI, G. et al. Antimicrobial potential of polyphenols extracted from almond skins. Letters in Applied Microbiology, v. 51, n. 1, p. 83-89, 2010. https://doi.org/10.1111/j.1472-765X.2010.02862.x.

MARTIN, J. G. P. et al. Antimicrobial potential and chemical composition of agroindustrial wastes. Journal of Natural Products, v. 5, p. 27-36, 2012.

MARTINI, S. et al. Antimicrobial activity against Helicobacter pylori strains and antioxidant properties of blackberry leaves (Rubus ulmifolius) and isolated compounds. International Journal of Antimicrobial Agents, v. 34, n. 1, p. 50-59, 2009. https://doi.org/10.1016/j.ijantimicag.2009.01.010.

MATTILA, P. H. et al. Polyphenol and vitamin C contents in European commercial blackcurrant juice products. Food Chemistry, v. 127, n. 3, p. 1216-1223, 2011. https://doi.org/10.1016/j.foodchem.2011.01.129.

O'SHEA, N.; ARENDT, E. K.; GALLAGHER, E. Dietary fibre and phytochemical characteristics of fruit and vegetable by-products and their recent applications as novel ingredients in food products. Innovative Food Science and Emerging Technologies v.16, p.1-10, 2012. https://doi.org/10.1016/j.ifset.2012.06.002.

OSAILI, T. M. et al. Effect of active essential oils added to chicken tawook on the behaviour of Listeria monocytogenes, Salmonella spp. and Escherichia coli O157:H7 during storage. International Journal of Food Microbiology, v. 337, p. 108947, 2021. https://doi.org/10.1016/j.ijfoodmicro.2020.108947.

PERUMALLA, A. V. S.; HETTIARACHCHY, N. S. Green tea and grape seed extracts - Potential applications in food safety and quality. Food Research International, v. 44, n. 4, p. 827-839, 2011. https://doi.org/10.1016/j.foodres.2011.01.022. 
RIBEIRO, J. S. et al. Natural antioxidants used in meat products: A brief review. Meat Science, v.148, p. 181-188, 2019. https://doi.org/10.1016/j.meatsci.2018.10.016.

RUBILAR, M. et al. Separation and HPLC-MS identification of phenolic antioxidants from agricultural residues: Almond hulls and grape pomace. Journal of Agricultural and Food Chemistry, mv. 55, n. 25, p. 10101-10109, 2007. https://doi.org/10.1021/jf0721996.

SAGAR, N. A. et al. Fruit and vegetable waste: Bioactive compounds, their extraction, and possible utilization. Comprehensive Reviews in Food Science and Food Safety, v. 17, n. 3, p. 512-531, 2018. https://doi.org/10.1111/1541-4337.12330.

SAGDIC, O. et al. RP-HPLC-DAD analysis of phenolic compounds in pomace extracts from five grape cultivars: Evaluation of their antioxidant, antiradical and antifungal activities in orange and apple juices. Food Chemistry, v. 126, n. 4, p. 1749-1758, 2011. https://doi.org/10.1016/j.foodchem.2010.12.075.

SALAS, M. P. et al. Antifungal activity of natural and enzymatically-modified flavonoids isolated from citrus species. Food Chemistry, v. 124, n. 4, p. 1411-1415, 2011. https://doi.org/10.1016/j.foodchem.2010.07.100.

SÁNCHEZ-GONZÁLEZ, L. et al. Antimicrobial activity of polysaccharide films containing essential oils. Food Control, v. 22, n. 8, p. 1302-1310, 2011. https://doi.org/10.1016/j.foodcont.2011.02.004.

SERGENT, T. et al. Anti-inflammatory effects of dietary phenolic compounds in an in vitro model of inflamed human intestinal epithelium. Chemico-Biological Interactions, v. 188, n. 3, p. 659-667, 2010. https://doi.org/10.1016/j.cbi.2010.08.007.

SHIEKH, K. A.; BENJAKUL, S.; SAE-LEAW, T. Effect of Chamuang (Garcinia cowa Roxb.) leaf extract on inhibition of melanosis and quality changes of Pacific white shrimp during refrigerated storage. Food Chemistry, v. 270, p. 554-561, 2019. https://doi.org/10.1016/j.foodchem.2018.07.139.

SUDJANA, A. N. et al. Antimicrobial activity of commercial Olea europaea (olive) leaf extract. International Journal of Antimicrobial Agents, v. 33, n. 5, p. 461-463, 2009. https://doi.org/10.1016/j.ijantimicag.2008.10.026.

SZABO, M. R. et al. Antioxidant and antimicrobial properties of selected spice extracts. International Journal of Food Properties, v. 13, n. 3, p. 535-545, 2010. https://doi.org/10.1080/10942910802713149.

SZKUDELSKA, K.; SZKUDELSKI, T. Resveratrol, obesity and diabetes. European $\begin{array}{llllll}\text { Journal of } & \text { Pharmacology, } & \text { v.635, }\end{array}$ https://doi.org/10.1016/j.ejphar.2010.02.054.

TAVEIRA, M. et al. Lycopersicon esculentum Seeds: An industrial byproduct as an antimicrobial agent. Journal of Agricultural and Food Chemistry, v. 58, n. 17, p. 9529-9536, 2010. https://doi.org/10.1021/jf102215g.

TIWARI, B. K. et al. Application of natural antimicrobials for food preservation. Journal of Agricultural and Food Chemistry, v. 57, p. 5987-60000, 2009. https://doi.org/10.1021/jf900668n.

TREMOCOLDI, M. A. et al. Exploration of avocado by-products as natural sources of bioactive compounds. PLOS ONE, v. 13, n. 2, p. e0192577, 2018. 
https://doi.org/10.1371/journal.pone.0192577.

VAN DER GOOT, A. J. et al. Concepts for further sustainable production of foods. Journal of Food Engineering, v.168, p. 42-51, 2016. https://doi.org/10.1016/j.jfoodeng.2015.07.010.

WANG, J. G. et al. The effect of cinnamon extract on insulin resistance parameters in polycystic ovary syndrome: a pilot study. Fertility and Sterility, v. 88, n. 1, p. 240-243, 2007. https://doi.org/10.1016/j.fertnstert.2006.11.082.

WHO. Food safety. World Health Organizations. 2020. Disponível em: https://www.who.int/en/news-room/fact-sheets/detail/food-safety. Acesso em: 14 nov. 2020.

WONGHIRUNDECHA, S.; SUMPAVAPOL, P.. Antibacterial activity of selected plant by-products against food-borne pathogenic bacteria. In. INTERNATIONAL CONFERENCE ON NUTRITION AND FOOD SCIENCES 2012, Anais [...]. [s.I: s.n.] p. 116-120. Disponível em: http://www.ipcbee.com/vol39/023-ICNFS2012-N038.pdf. Acesso em: 19 mar. 2017.

YAHIA, E. M. Fruit and vegetable phytochemicals. Chichester: Wiley-Blackwell Publishing, 2017. 1488p.

ZINOVIADOU, K. G.; KOUTSOUMANIS, K. P.; BILIADERIS, C. G. Physico-chemical properties of whey protein isolate films containing oregano oil and their antimicrobial action against spoilage flora of fresh beef. Meat Science, v. 82, n. 3, p. 338-345, 2009. https://doi.org/10.1016/j.meatsci.2009.02.004.

\section{Autores}

Nathana Lazzarotto Cristofoli ${ }^{1}$, Francine Novack Victoria ${ }^{2, *}$

1. Curso de Pós-Graduação Lato Sensu em Ciência dos Alimentos, Centro de Ciências Químicas, Farmacêuticas e de Alimentos, Universidade Federal de Pelotas, Campus Capão do Leão, Caixa Postal 354, CEP. 96010-900, Pelotas, RS, Brasil; Universidade do Algarve, Campus da Penha, 8005-139, Faro, Portugal.

2. Centro de Ciências Químicas, Farmacêuticas e de Alimentos, Universidade Federal de Pelotas, Campus Capão do Leão, Caixa Postal 354, CEP. 96010-900, Pelotas, RS, Brasil.

* Autor para correspondência: francinevictoria@yahoo.com.br 